VA; the State Laboratory Institute, Massachusetts Department of Public Health; Massachusetts General Hospital, Boston; the Lincoln Clinic, NB; and the Division of Human Genetics, University of Maryland School of Medicine, Baltimore. Twelve boys and four girls identified by newborn screening had partial biotinidase deficiency, defined as $10 \%$ to $30 \%$ of the mean normal activity. Three siblings of these children also had partial deficiency. Fourteen children ascertained by neonatal screening had profound biotinidase deficiency (less than 108 mean normal activity). Two siblings with profound deficiency were found among older siblings of these children. In 24 children with symptoms biotinidase activity levels were less than $10 \%$ of the mean normal level. All children with partial deficiency were healthy at the time of diagnosis. One child not treated initially with biotin later developed hypotonia, hair loss and skin rash which resolved with biotin therapy. Delayed development of symptams in some cases may depend on the interaction of reduced biotinidase activity with other factors. e.g. availability of exogenous biotin and alterations in metabolic demand for the vitamin. The need for biotin supplementation may be increased at times of infection and stress. (McVoy JRS, Wolf B et al. Partial biotinidase deficiency: Clinical and biochemical features. $J$ Pediatr Jan $1990 ; 116: 78-83)$.

COMMENT. Biotin responsive late onset multiple carboxylase deficiency is an autosamal recessive inherited disorder manifested by seizures, alopecia, skin rash, hypotonia, ataxia, hearing loss, and developmental delay. Lactic acidosis and organic aciduria are often present and if untreated the symptams become progressively worse and cama and death may occur. Symptams of biotinidase deficiency resolve rapidly after treatment with biotin 5-10 mg daily orally, but neurologic damage may be irreversible. Early diagnosis and treatment of partial biotinidase deficiency may prevent the development of potentially serious consequences.

\title{
DEGENERATIVE DISORDERS
}

\section{ATAXIA-TEIANGIECTASIA}

The proportion of T-cell antigen receptors in ten patients with ataxia-telangiectasia were compared with normal subjects and patients with other immune deficits at the Departments of Clinical Immunology and Pediatrics, University of Rome, "la Sapienza" Rome, Italy. An increased ratio of ganma/delta bearing to alpha/beta bearing T-cells in ataxia-telangiectasia may reflect both a recombinational defect that interferes with $\mathrm{T}$-cell and $\mathrm{B}-\mathrm{cell}$ gene rearrangements and an inability to repair damage to the DNA. The criteria for the diagnosis of ataxia-telangiectasia included early cerebellar ataxia, chromosamal instability, and raised alpha fetoprotein levels. There were six boys and four girls ranging in age from 2-18 years. The diagnos is was confirmed by cytogenetic analysis which showed the typical increase in 
nonrandom chromosomal breaks and translocations. (Carbonari M et al. Relative increase of $\mathrm{T}$ cells expressing the gamma/delta rather than the alpha/beta receptor in ataxia-telangiectasia. N Engl J Med Jan $11,1990 ; 322: 73-76)$.

COMMENT. In an editorial in the same issue Peterson RDA and Funkhouser JD of the University of South Alabama, Mobile, AL refer to their previously published proposal that essentially all clinical manifestations of ataxia-telangiectasia, including the degeneration of the CNS, are a consequence of a defect in genetic recombination. (Immunol Today 1989; 10:313-5). Elucidation of the molecular abnormalities of the lymphocytes in patients with ataxia-telangiectasia may reveal molecular mechanisms responsible for the cellular differentiation of lymphocytes and other cell systems. The gene responsible for ataxia-telangiectasia has been localized to chromosome 1lq22-23. These studies at the molecular level bring new insight in lymphocyte differentiation and the immune disorder that characterizes ataxia-telangiecasia. Absence of tonsils in a child with ataxia should prompt the determination of immunoglobulins.

\section{METACHROMATIC LEUKODYSTROPHY}

A ten year old girl with metachromatic leukodystrophy in whom neurophysiologic function and sulfatide metabolism had improved after she received a bone marrow transplant five years before is reported from the Bone Marrow Transplantation Program, Department of Pediatrics and Division of Pediatric Neurology, University of Minnesota, MN and other centers. The diagnosis was confirmed by enzyme analys is at eight months of age after an older sister had been found to have the disease. Serial MRI of the head obtained before and after bone marrow transplantation showed no further deterioration of white matter. Sural nerve specimens obtained by biopsy before and two years after transplantation showed less accumulation of lipid in the macrophages on electron microscopy. Sulfatide levels in the CSF were within normal limits at seven and ten years of age. Asymptomatic infants, children and young adolescents who are found to have the disease after it has been diagnosed in an older sibling should be considered for bone marrow transplantation. (Krivit $W$ et al. Treatment of late infantile metachromatic leukodystrophy by bone marrow transplantation. N Engl J Med Jan 4,$1990 ; 322: 28-32)$.

COMMENT. Dr. John Menkes gives an excellent overview of the leukodystrophies in an editorial in this issue (N Engl J Med Jan 4, 1990; 322:34-35). Metachromat ic leukodyst rophy and adrenoleukodystrophy may be amenable to new experimental therapies. No treatment is available for globoid-cell, Canavan, Pelizaeus-Merzbacher, and Alexander varieties of leukodystrophy. 